\title{
TWO-DIMENSIONAL ERROR CONTROL BASED ON MODULAR CORRECTING CODES
}

\author{
Jürgen Sieck ${ }^{1)}$, Vasyl Yatskiv ${ }^{2)}$, Anatoly Sachenko ${ }^{2)}$, Taras Tsavolyk ${ }^{2)}$ \\ ${ }^{1)}$ University of Applied Sciences Berlin \\ Wilhelminenhofstr.75A, 12459 Berlin, Germany, j.sieck@htw-berlin.de \\ ${ }^{2)}$ Ternopil National Economic University, 3 Peremohy Square, Ternopil, Ukraine, 46020, \\ vy@tneu.edu.ua, as@tneu.edu.ua
}

\begin{abstract}
The paper presents a method of detecting and correcting packet errors in the block of data based on the modular corrective codes. Check symbols are calculated separately in rows and columns of the data matrix. Herewith, the same data matrix coefficients are used for calculating the check symbols in rows and columns. This allows the detection and correction of errors packets that are in the same row or column. When two or more distorted information symbols are in the same row (assuming that there is only one error in the column) then errors can be corrected through the analysis of the column syndrome. The possible cases of the distorted symbols placement in a block of data and ways of their fixing are considered. The algorithm for detecting and correcting errors packets is elaborated. In the general case the offered method of error correction, based on modular correcting code, provides a correction of: $\mathrm{n}$ errors, which are in the same row or column of the data matrix of $\mathrm{n}$ size; $2 \cdot n-1$ errors that are in the same row and column. The proposed method of encoding / decoding is designed in Verilog and implemented on FPGA in the Quartus II of Altera company. Copyright $(\mathcal{C}$ Research Institute for Intelligent Computer Systems, 2015. All rights reserved.
\end{abstract}

Keywords: wireless sensor networks, encoding, decoding, modular correcting codes, residual number system.

\section{INTRODUCTION}

Nowadays with the development and widespread implementation of wireless technologies, the improvement of reliability of data transmission is becoming increasingly important. In accordance with this, the control of data integrity, which is transmitted and processed by modern digital systems, is an actual scientific problem especially for the area of wireless sensor networks (WSN) [1].

While providing the required level of WSN reliability, in contrast to the wireless computer networks, it is necessary to take into account the limitations of computing resources of computer units and the use of autonomous power. In addition, packages retransmission mode, which is used in most WSN applications, causes extra complications.

Whereas the techniques of traffic redundancy reduction by data compressing, correlated data filtering and excluding of sensors redundant data being applied for WSN [2], the demands for reliable transmission of each data package correspondingly increase.

The optimal correcting codes for the WSN were widely studied by the researchers in the last decade $[1,3-5]$, but the type of error correcting codes has not been standardized yet.
There are two main types of error correcting codes, block codes and convolutional codes. Among the most common block codes, the following ones can be mentioned: Hemet Golay (Golay) codes, Bouza-Chadhuri-Hocquenghem (BCH), ReedSolomon code and low density parity code (LDPC code), which also refers to the class of block codes [6].

$\mathrm{BCH}$ code, Reed-Solomon code and convolutional code were studied in [7] from the perspective of implementation complexity and power consumption on FPGA and applicationspecific integrated circuits (ASIC). The results show that convolutional codes have advantages over $\mathrm{BCH}$ and Reed-Solomon codes, but at the same time Viterbi decoder consumes more energy than the uncoded data transmission. Thus, convolutional codes are not very suitable for self-powered WSN nodes. This indicates that the binary $\mathrm{BCH}$ code, implemented on ASIC, is the better alternative for WSN that work in open space.

The results, obtained in [8], show that energy efficiency of ARQ mechanism does not depend on package retransmission, but depends on the size of the package and the distance between nodes.

In [9] the authors suggest using of low density parity check codes both for channel coding and 
source coding to reduce power consumption in WSN. The results of modeling show that using of LDPC codes increases energy costs by $42 \%$ compared to the $\mathrm{BCH}$ codes.

In [10] the adaptive scheme is proposed, where the output node has the ability to change the error correction circuit for each transmission. The results of studies of energy efficiency of adaptive and nonadaptive error correction schemes with different distances and sizes of communications packages show that the adaptive scheme has a higher energy efficiency compared to non-adaptive one with all the distances and sizes of packages.

In [11] Reed-Solomon (RS) codes were used for a source node coding and decoding only at the base station to save energy at the node level, and thereby to extend the network.

The study of energy costs for different modulation schemes and Reed-Solomon codes of different lengths $[12,13]$ has shown that ReedSolomon code with parameters $(63,59,5)$ is the most suitable compared to the transmission without coding. In [14] a scheme is proposed in which LDPC-code is used for data encoding in the transmitting node and the partial decoding is performed in the intermediate nodes and full decoding is performed only at the base station.

Performance evaluation of the use of various error-correcting codes [1] shows that stronger codes provide better performance, but they are energy inefficient in comparison with simple codes. The latter have lower performance but are more energy efficient.

A strategy of error control (node - node, from end-to-end) should be taken into account while selecting a scheme of error control in WSN. Powerful codes are the most suitable for the use of error control scheme from end to end while simple codes are optimal to control errors in each node.

As it is shown above, many studies prove the benefits of the use of WSN Reed - Solomon codes $[1,15]$. However, Reed-Solomon codes make impossible to use adaptive error control schemes, as all check symbols should be calculated when there is an increase / decrease in the number of check symbols [16].

Due to the limited functional characteristics of wireless sensors, the selection of correcting codes for WSN is considered an actual problem. Thus, correcting codes for WSN should meet the following needs:

- low complexity of coding / decoding algorithms;

- low hardware requirements to the algorithms implementation (microcontroller capacity and clock speed, memory capacity);

- adaptive change of a number of check symbols due to the changes of the channel parameters.

\section{CORRECTING CODES OF RESIDUAL NUMBER SYSTEM}

It should be noted that processing and transmission of data in the residual number system (RNS) has certain advantages due to the equality between residues, their independence, low capacity and possibility of parallel execution of arithmetic operations. Due to these advantages of RNS while data processing and transmission, corrective codes, based on modular arithmetic, is of particular interest for the use in WSN $[17,18]$.

In addition to the mentioned above advantages of RNS, the effective correcting codes, which are able to detect and correct errors packages were also developed $[18,19]$. However, in order to use them in the existing digital systems of data transmission, including WSN, you must first convert RNS data that requires additional effort and computing resources.

Let's consider the residual number system with modules $p_{1}, p_{2}, \ldots, p_{i}, \ldots, p_{n}$ where number $A$ is represented by a set of residues due to the corresponding modules

$$
A=\left(b_{1}, b_{2}, \ldots, b_{i}, \ldots, b_{n}\right),
$$

where $b_{i}=A\left(\bmod p_{i}\right)$.

Modules $p_{i}$ are selected under the condition that the greatest common divisor is $\operatorname{gcd}\left(p_{i}, p_{j}\right)=1$, at $i \neq j$, a $p_{1}<p_{2}<\ldots p_{i} \ldots<p_{n}$.

From this set of modules the first $k$ of modules are used for data encoding and $r=n-k$ of modules are used to detect and correct errors. A set of $k$ modules forms a working range:

$$
P=\prod_{i=1}^{k} p_{i},
$$

and a total range is:

$$
\wp=P \cdot \prod_{i=1}^{n-k} p_{i}=\prod_{i=1}^{n} p_{i} .
$$

In correcting codes RNS (R - RNS codes) the value of the redundant check symbol [18] is calculated due to

$$
b_{k+1}=A\left(\bmod p_{k+1}\right),
$$

i.e. while calculating the value of the redundant check symbol you need to return to the value of the 
original $A$, and this requires additional computing resources.

R-RNS codes have also certain constraints, depending on the selection of the increasing sequence of relatively prime modules. Thus, all test modules must be identical in capacity. Otherwise, the problem of efficient (optimal) storage of check symbols arises.

In [19] weak arithmetic codes (WA - RNS codes) were developed which have certain constraints while controlling arithmetic operations. However, these codes can be effectively used to detect and correct errors during data transfer, as they allow to form check symbols easily.

The value of the redundant check symbol in weak arithmetic codes RNS is [19]

$$
\bar{b}_{k+1}=\left(\begin{array}{l}
v_{1} \cdot b_{1}+v_{2} \cdot b_{2}+\ldots+ \\
+\ldots+v_{i} \cdot b_{i}+\ldots+v_{k} \cdot b_{k}
\end{array}\right)(\bmod p),
$$

where $v_{i}$ - relatively prime coefficients of $p ; b_{i}$ residues of the relevant modules $p_{i}, i=1, \ldots, k$.

Suppose that in the process of transmission error occurred in one of the residues and $A^{\prime}$ is received instead of $A$ :

$$
A^{\prime}=\left(b_{1}, b_{2}, \ldots, b_{i}^{\prime}, \ldots, b_{k}, \bar{b}_{k+1}\right) .
$$

The value of the redundant check symbol is calculated due to the formula

$$
\bar{b}_{k+1}^{\prime}=\left(\begin{array}{l}
v_{1} \cdot b_{1}+v_{2} \cdot b_{2}+\ldots+ \\
+\ldots+v_{i} \cdot b_{i}^{\prime}+\ldots+v_{k} \cdot b_{k}
\end{array}\right)(\bmod p) .
$$

Then, the difference between the calculated and adopted check symbols is calculated as $\delta=\bar{b}_{k+1}^{\prime}-b_{k+1}$. Thus, there is no error, if $\delta=0$; and we have an error, if $\delta \neq 0$.

To detect an error in any of the units, it is necessary that each value of $\delta_{i}$ would correspond to one error value of $e_{i}$. To satisfy this condition, the coefficients $v_{i}$ should be relatively prime to $p$.

The disadvantage of weak arithmetic RNS codes is that the data, which has to be processed or transmitted, should be presented in RNS and this limits its use.

In [20] modular correcting codes ( $\mathrm{M}$ - codes), which have the advantages of weak arithmetic codes, but process input data, represented in a positional number system (binary, octal, decimal) that extends their scope, have been developed and researched. However, to correct error packages, it is necessary to calculate two or more check symbols and this causes an increase of redundancy code.

\section{THE METHOD OF TWO-DIMENSIONAL ERROR CONTROL}

The authors proposed correcting codes, based on modular arithmetic, which provide two-dimensional error control. Two-dimensional control refers to the formation of check symbols in rows and columns of data matrix.

Check symbol in a row:

$$
x_{i, k+1}=\left(\begin{array}{c}
v_{1} \cdot x_{i, 1}+v_{2} \cdot x_{i, 2}+\ldots+ \\
+\ldots+v_{i} \cdot x_{i, i}+\ldots+v_{k} \cdot x_{i, k}
\end{array}\right)(\bmod p),
$$

where $v_{i}$ - relatively prime numbers with $p, p-$ test module, $x_{i}$ - information symbols of a given capacity, represented in a positional number system.

The value of the test module is calculated under the condition [20]:

$$
p \geq 2 \cdot n \cdot\left(2^{m}-1\right)
$$

where $m$ - capacity of information symbol, $k-$ a number of information symbols.

Check symbol in a column:

$x_{k+2, j}=\left(\begin{array}{l}v_{1} \cdot x_{1, j}+v_{2} \cdot x_{2, j}+\ldots+ \\ +\ldots+v_{i} \cdot x_{i, j}+\ldots+v_{k} \cdot x_{k, j}\end{array}\right)(\bmod p)$.

Two-dimensional error control can be presented as a matrix:

$$
\begin{array}{cccccc|l}
x_{1,1} & x_{1,2} & \ldots & x_{1, j} & \ldots & x_{1, k} & x_{1, k+1} \\
x_{2,1} & x_{2,2} & \ldots & x_{2, j} & \ldots & x_{2, k} & x_{2, k+1} \\
\ldots & \ldots & \ldots & \ldots & \ldots & \ldots & \ldots \\
x_{i, 1} & x_{i, 2} & \ldots & x_{i, j} & \ldots & x_{i, k} & x_{i, k+1} \\
\ldots & \ldots & \ldots & \ldots & \ldots & \ldots & \ldots \\
x_{k, 1} & x_{k, 2} & \ldots & x_{k, j} & \ldots & x_{k, k} & x_{k, k+1} \\
x_{k+2,1} & x_{k+2,2} & \ldots & x_{k+2, j} & \ldots & x_{k+2, k} &
\end{array}
$$

On the receiving side, the check symbols $x_{i, k+1}^{\prime}$ and $x_{k+2, j}^{\prime}$ for the received data are calculated according to the decoder formulas (1), (3). As a result, we obtain a matrix calculation: 


$$
\begin{array}{cccccc|l}
x_{1,1} & x_{1,2} & \ldots & x_{1, j}^{\prime} & \ldots & x_{1, k} & x_{1, k+1}^{\prime} \\
x_{2,1} & x_{2,2} & \ldots & x_{2, j}^{\prime} & \ldots & x_{2, k} & x_{2, k+1}^{\prime} \\
\ldots & \ldots & \ldots & \ldots & \ldots & \ldots & \ldots \\
x_{i, 1} & x_{i, 2} & \ldots & x_{i, j}^{\prime} & \ldots & x_{i, k} & x_{i, k+1}^{\prime} \\
\ldots & \ldots & \ldots & \ldots & \ldots & \ldots & \ldots \\
x_{k, 1} & x_{k, 2} & \ldots & x_{k, j}^{\prime} & \ldots & x_{k, k} & x_{n, k+1}^{\prime} \\
x_{k+2,1}^{\prime} & x_{k+2,2}^{\prime} & \ldots & x_{k+2, j}^{\prime} & \ldots & x_{k+2, k}^{\prime} &
\end{array}
$$

Error detection occurs according to the analysis of the syndrome, which is calculated due to the formula

$$
\delta_{i, j}=\left(x_{i, j}^{\prime}-x_{i, j}\right) \bmod p
$$

or

$$
\begin{gathered}
\delta_{i}=\left|\begin{array}{c}
\left(x_{1, k+1}^{\prime}-x_{1, k+1}\right) \bmod p \\
\left(x_{2, k+1}^{\prime}-x_{2, k+1}\right) \bmod p \\
\ldots \\
\left(x_{i, k+1}^{\prime}-x_{i, k+1}\right) \bmod p \\
\ldots \\
\left(x_{k, k+1}^{\prime}-x_{k, k+1}\right) \bmod p
\end{array}\right|, \\
\delta_{j}=\left|\begin{array}{c}
\left(x_{k+2,1}^{\prime}-x_{k+2,1}\right) \bmod p \\
\left(x_{k+2,2}^{\prime}-x_{k+2,2}\right) \bmod p \\
\left(x_{k+2, j}^{\prime}-x_{k+2, j}\right) \bmod p \\
\ldots \\
\left(x_{k+2, k}^{\prime}-x_{k+2, k}\right) \bmod p
\end{array}\right|,
\end{gathered}
$$

where $x_{i, k+1}^{\prime}$ - check symbols, calculated according to the received data.

So, there is no error, if $\delta_{i, j}=0$, then the syndrome

$$
\delta_{i}=\left|\begin{array}{c}
0 \\
0 \\
\ldots \\
0 \\
\cdots \\
0
\end{array}\right| \text { and } \delta_{j}=\left|\begin{array}{c}
0 \\
0 \\
\cdots \\
0 \\
\cdots \\
0
\end{array}\right| \text {-there is no error. }
$$

When $\delta_{i, j} \neq 0$, an error occurs at the intersection of the appropriate row and column. Thus, non-zero value of the syndrome clearly points to the distorted information symbol.
When two or more data symbols are distorted in the same raw (providing that there is one error in a column), an error is corrected according to the analysis of the syndrome of the appropriate column.

Similarly, if two or more data symbols, located in the same column, are distorted, then an error is corrected according to the analysis of the syndrome of the appropriate row.

To correct data symbols errors, located in $n-1$ row and in $n-1$ column, it is necessary to solve the equation:

$$
\begin{aligned}
& \left(v_{1} \cdot\left(x_{\mathrm{i}, 1}^{\prime}-x_{\mathrm{i}, 1}\right)+v_{2} \cdot\left(x_{\mathrm{i}, 2}^{\prime}-x_{\mathrm{i}, 2}\right)+\ldots+\right. \\
& +\ldots+v_{i} \cdot\left(x_{i, j}^{\prime}-x_{\mathrm{i}, j}\right)+\ldots+ \\
& \left.+\ldots+v_{n} \cdot\left(x_{i, k}^{\prime}-x_{\mathrm{i}, k}\right)\right) \bmod p=\delta_{i, 1}
\end{aligned}
$$

If the greatest common divisor $\operatorname{gcd}\left(v_{i}, p\right)=1$, i.e. $\left(\mathrm{v}_{i}, p\right)$ are relatively prime numbers, then the equation (4) has a unique solution that will match the correct information symbols.

\section{EXPERIMENTAL STUDIES AND IMPLEMENTATION OF THE METHOD}

Let's consider, for example, transmission of data, which is formed in a matrix of $k \times k$ size, when $k=8, m=8$ where $k$ is the dimension of data matrix, $m$ is data capacity.

$$
\begin{array}{llllllll|l}
x_{1,1} & x_{1,2} & x_{1,3} & x_{1,4} & x_{1,5} & x_{1,6} & x_{1,7} & x_{1,8} & x_{1,9} \\
x_{2,1} & x_{2,2} & x_{2,3} & x_{2,4} & x_{2,5} & x_{2,6} & x_{2,7} & x_{2,8} & x_{2,9} \\
x_{3,1} & x_{3,2} & x_{3,3} & x_{3,4} & x_{3,5} & x_{3,6} & x_{3,7} & x_{3,8} & x_{3,9} \\
x_{4,1} & x_{4,2} & x_{4,3} & x_{4,4} & x_{4,5} & x_{4,6} & x_{4,7} & x_{4,8} & x_{4,9} \\
x_{5,1} & x_{5,2} & x_{5,3} & x_{5,4} & x_{5,5} & x_{5,6} & x_{5,7} & x_{5,8} & x_{5,9} . \\
x_{6,1} & x_{6,2} & x_{6,3} & x_{6,4} & x_{6,5} & x_{6,6} & x_{6,7} & x_{6,8} & x_{6,9} \\
x_{7,1} & x_{7,2} & x_{7,3} & x_{7,4} & x_{7,5} & x_{7,6} & x_{7,7} & x_{7,8} & x_{7,9} \\
x_{8,1} & x_{8,2} & x_{8,3} & x_{8,4} & x_{8,5} & x_{8,6} & x_{8,7} & x_{8,8} & x_{8,9} \\
x_{9,1} & x_{9,2} & x_{9,3} & x_{9,4} & x_{9,5} & x_{9,6} & x_{9,7} & x_{9,8} &
\end{array}
$$

With the help of the developed software the values of the relatively prime coefficients $v_{i}$ and a test module $p$ have been experimentally found: $p=131071, \quad v_{1}=3, \quad v_{2}=257, \quad v_{3}=263$, $v_{4}=269, \quad v_{5}=271, \quad v_{6}=277, \quad v_{7}=281$, $v_{8}=283 . \quad \delta_{i j}=v_{j} \cdot x_{i}(\bmod p), 1 \leq j \leq 8$. 
Table 1 shows the value of the information symbols of 8 bits capacity, an syndrome $\delta_{i j}=v_{j} \cdot e_{i}(\bmod p), 1 \leq j \leq 8$, calcu- error value is in the range: $-255 \leq e_{i} \leq 255$. lated for all possible variants of $x_{i}$ errors. For

Table 1. Values of the syndrome for eight information symbols

\begin{tabular}{|c|c|c|c|c|c|c|c|c|}
\hline $\begin{array}{c}\text { Error } \\
\text { value: } \\
e_{i}\end{array}$ & 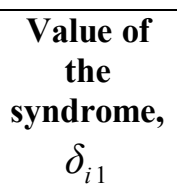 & 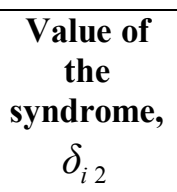 & 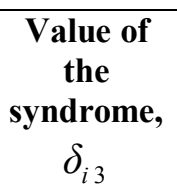 & 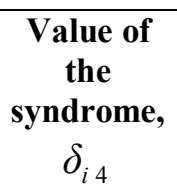 & 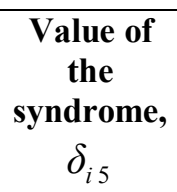 & 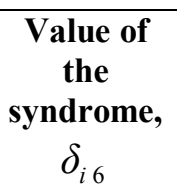 & 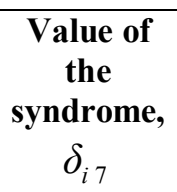 & 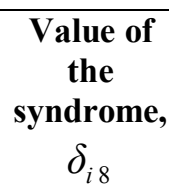 \\
\hline 1 & 3 & 257 & 263 & 269 & 271 & 277 & 281 & 283 \\
\hline 2 & 6 & 514 & 526 & 538 & 542 & 554 & 562 & 566 \\
\hline 3 & 9 & 771 & 789 & 807 & 813 & 831 & 843 & 849 \\
\hline 4 & 12 & 1028 & 1052 & 1076 & 1084 & 1108 & 1124 & 1132 \\
\hline$\ldots$ & & & & $\ldots$ & & & &.. \\
\hline 255 & 765 & 65535 & 67065 & 68595 & 69105 & 70635 & 71655 & 72165 \\
\hline-1 & 131068 & 130814 & 130808 & 130802 & 130800 & 130794 & 130790 & 130788 \\
\hline-2 & 131065 & 130557 & 130545 & 130533 & 130529 & 130517 & 130509 & 130505 \\
\hline-3 & 131062 & 130300 & 130282 & 130264 & 130258 & 130240 & 130228 & 130222 \\
\hline-4 & 131059 & 130043 & 130019 & 129995 & 129987 & 129963 & 129947 & 129939 \\
\hline ... & & & & & & & & $\cdots$ \\
\hline-255 & 130306 & 65536 & 64006 & 62476 & 61966 & 60436 & 59416 & 58906 \\
\hline
\end{tabular}

An encoding algorithm as a combination of the following steps has been developed on the basis of the proposed method:

1. Data, which has to be transmitted, form a matrix of $k \times k$ size.

2. Test module $p$ and relatively prime coefficients $v_{1}, \ldots, v_{i}, \ldots, v_{k}$ are selected.

3. Check symbols are calculated for the rows of the matrix according to the formula (1).

4. Check symbols are calculated for the columns of the matrix according to the formula (2).

An error detection algorithm as a combination of the following steps has also been developed:

1. Data, which has been obtained, form a matrix of $k \times k$ size.

2. Check symbols are calculated for the rows of the matrix according to the formula (1).

3. Check symbols are calculated for the columns of the matrix according to the formula (2).

4. A syndrome $\delta_{i, j}=\left(x_{i, j}^{\prime}-x_{i, j}\right) \bmod p$ is calculated for the rows and columns of the data matrix.

5. If $\delta_{i, j}=0$, then $x_{i, j}=x_{i, j}^{\prime}$, so there`s no error.

6. If $\delta_{i, j} \neq 0$, then an error is found.

When a single information symbol is distorted, then the syndrome value at the intersection of a row and a column is not zero.

Let's consider the probabilities of information symbols distortion.
1. The syndrome is not zero only for one row and column. A symbol is distorted at the intersection of the appropriate row and column. Errors are corrected according to the table of the syndromes (Table 1).

2 . The value of the syndrome $\delta_{i, j} \neq 0$ for two or more rows and one column. Information symbols, which are in the same column, were distorted. Errors are corrected according to the table of the syndromes (Table 1).

3 . The value of the syndrome $\delta_{i, j} \neq 0$ for two or more columns and one row. Information symbols, which are in the same row, were distorted. Errors are corrected according to the table of the syndromes (Table 1).

4. The value of the syndrome $\delta_{i, j} \neq 0$ for two or more rows and columns. The equation:

$$
\begin{aligned}
& \left(\mathrm{v}_{1} \cdot\left(\mathrm{x}_{1,1}^{\prime}-\mathrm{x}_{1,1}\right)+\mathrm{v}_{2} \cdot\left(\mathrm{x}_{1,2}^{\prime}-\mathrm{x}_{1,2}\right)+\ldots+\right. \\
& +\ldots+\mathrm{v}_{\mathrm{i}} \cdot\left(\mathrm{x}_{\mathrm{i}, \mathrm{j}}^{\prime}-\mathrm{x}_{\mathrm{i}, \mathrm{j}}\right)+\ldots+ \\
& \left.+\ldots+\mathrm{v}_{\mathrm{n}} \cdot\left(\mathrm{x}_{1, \mathrm{k}}^{\prime}-\mathrm{x}_{1, \mathrm{k}}\right)\right) \bmod \mathrm{p}=\delta_{1, j}
\end{aligned}
$$

must be solved for error correction.

Suppose that four symbols, located at the intersection of two rows and two columns, are distorted.

At first, suppose that errors occurred in two symbols, located in the first row. As there is no error $x_{i, j}^{\prime}-x_{i, j}=0$, the equation (5) can be written as: 


$$
\left(v_{1} \cdot\left(x_{1,1}^{\prime}-x_{1,1}\right)+v_{2} \cdot\left(x_{1,2}^{\prime}-x_{1,2}\right)\right) \bmod p=\delta_{1,1} .
$$

As the coefficients $v_{i}$ and $p$ are relatively prime numbers, then the equations (6) has a unique solution in integers. It should be noted that the found solutions match the corrected values of the information symbols $x_{1,1}, x_{1,2}$.

Having put $x_{1,1}, x_{1,2}$ into equation (5), check the value of the syndrome $\delta_{i, j}$. Thus, the following options are possible: 1$)$ the first row syndrome $\delta_{1,1}=0$ - errors are corrected; 2) the first column syndrome $\delta_{2,1} \neq 0$. Then, in Table 1 we find an error, matching the syndrome $\delta_{2,1}$, and correct it in the following way: $x_{2, i}=\delta_{2,1}-e_{i}$.

In case an assumption is wrong, check the following group of symbols.

Data encryption algorithm hardware complexity has been compared, while implementing FPGA encoders, correcting RNS codes and the developed modular correcting codes (MCC) with such parameters as: input symbols capacity - 8 bits; a number of information symbols $-2 \div 8$ bits (Fig. 1).

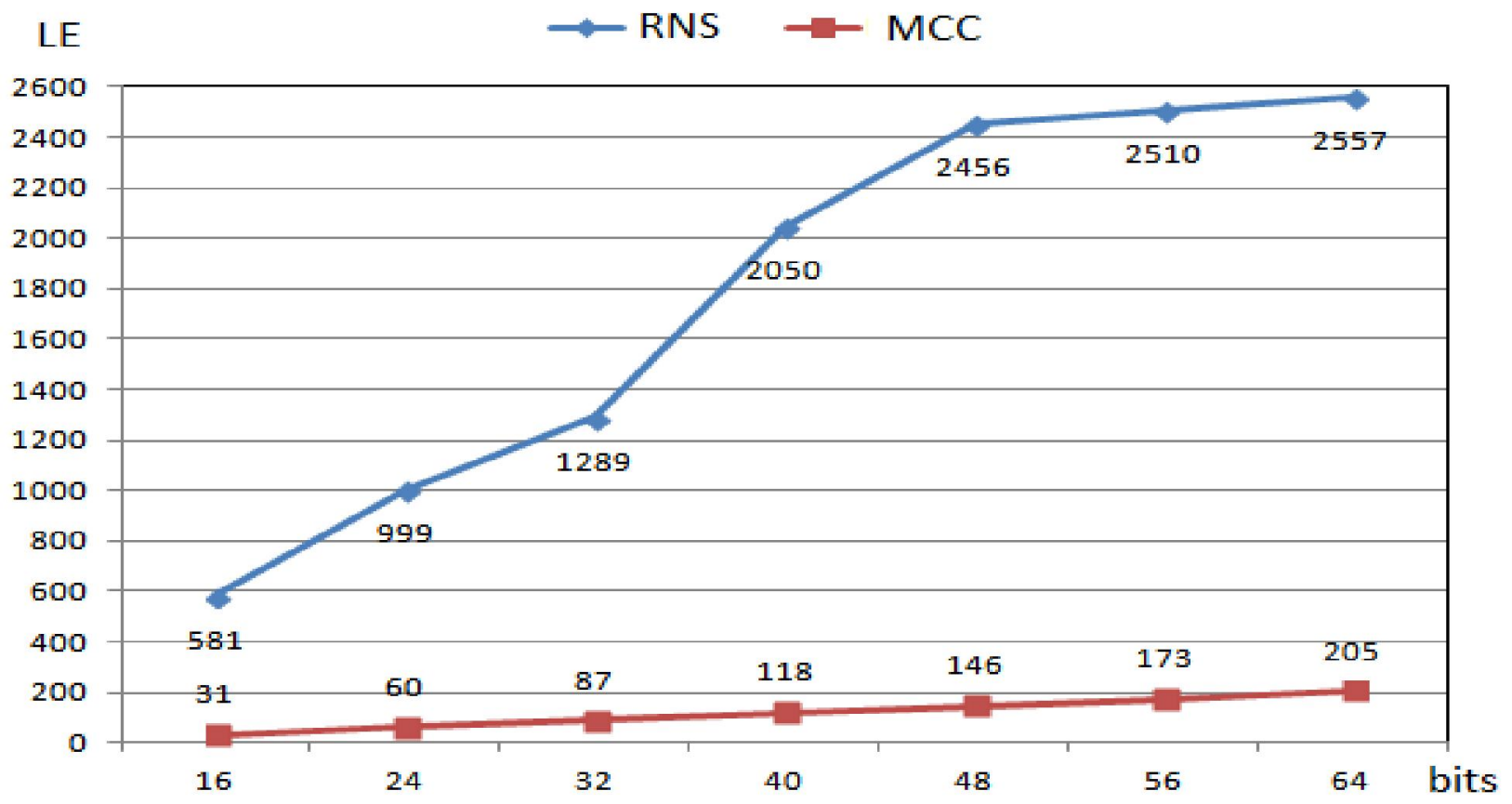

Fig. 1 - The hardware encoder complexity depending on the message capacity.

Fig. 1 shows that hardware encoding complexity of modular correcting codes is on average 16 times less than the corrective RNS codes.

Due to the low hardware complexity of the noiseimmune data encoding algorithm, the developed two-dimensional error control could be applied in wireless sensor networks, used in high electromagnetic interference environments [21].

\section{CONCLUSIONS}

The proposed two-dimensional error control, based on modular correcting codes, provides detection and correction of $k$ errors that are in the same row or column of the data matrix of $k \times k$ size, $2 \cdot k$ errors, provided that they are in two rows or two columns of the data matrix. Thus, the hardware complexity of modular correcting codes encoding on average is 16 times less than the corrective RNS codes.

\section{REFERENCES}

[1] S. L. Howard, C. Schlegel, K. Iniewski, Error control coding in low-power wireless sensor networks: When is ECC energy-efficient? EURASIP Journal on Wireless Communications and Networking, (2006) 1 (2006), pp. 1-14.

[2] T.Srisooksai, K. Keamarungsi, P.Lamsrichan, K.Araki. Practical data compression in wireless sensor networks: A survey. Journal of Network and Computer Applications, 35(1) (2012), pp. 37-59.

[3] M. C. Vuran, I. F. Akyildiz, Error control in wireless sensor networks: a cross layer analysis, IEEE/ACM Transactions on Networking, (17) 4 (2009), pp. 1186-1199.

[4] Z. Tian, D.F. Yuan, Q.Q. Liang, Energy efficiency analysis of error control schemes in wireless sensor networks, in Proceedings of the 
IEEE International Wireless Communications and Mobile Computing Conference IWCMC'08, (2008), pp. 401-405.

[5] N. Abughalieh, K. Steenhaut, A. Nowé, Low power channel coding for wireless sensor networks, in Proceedings of the 17th IEEE Symposium on Communications and Vehicular Technology in the Benelux SCVT'2010, (2010), pp. 1-5.

[6] B. Sklar, Digital Communications: Fundamentals and Applications, 2nd Edition, Prentice Hall, 2001, 1104 p.

[7] B. Gopinath, et al., Performance analysis of error control codes for wireless sensor networks, in Proceedings of the Fourth IEEE International Conference on Information Technology ITNG'07, (2007), pp. 876-879.

[8] I. Chlamtac, C. Petrioli, J. Redi, Energyconserving go-back-N ARQ protocols for wireless data networks, in Proceedings of IEEE ICUPC'98, (Piscataway, NJ, USA, October 1998), vol. 2, pp. 1259-1263.

[9] J. Kang, B. Zhou, Z. Ding, S. Lin, LDPC coding schemes for error control in a multicast network, in Proceedings of the IEEE International Symposium on Information Theory ISIT'08, (2008), pp. 822-826.

[10] G. A. Al-suhail, K. W. Louis, T. Y. Abdallah, Energy efficiency analysis of adaptive error correction in wireless sensor networks, International Journal of Computer Science Issues, (9) 4 (2012), pp. 79-84.

[11] S. Chouhan, R. Bose, and M. Balakrishnan, Integrated energy analysis of error correcting codes and modulation for energy efficient wireless sensor nodes, IEEE Transactions on Wireless Communications, (8) 10 (2009), pp. 5348-5355.

[12] S. Bin Qaisar, S. Karande, K. Misra, H. Radha, Optimally mapping an iterative channel decoding algorithm to a wireless sensor network, in Proceedings of the IEEE International Conference on Communications ICC'07, (2007), pp. 3283-3288.

[13] S. Srivastava, C. Spagnol, E. Popovici, Analysis of a set of error correcting schemes in multi-hop wireless sensor networks, in Research in Microelectronics and Electronics, PRIME'2009, (2009), pp. 1-4.

[14] M. Sartipi, F. Fekri, Source and channel coding in wireless sensor networks using LDPC codes, in Proceedings of the IEEE International Conference on Sensor and Ad Hoc
Communications and Networks SECON'2004, (2004), pp. 309-316.

[15] N. A. Alrajeh et al., Error correcting codes in wireless sensor networks: an energy perspective, Appl. Math, (9) 2 (2015), pp. 809818.

[16] V. T. Goh, M. U. Siddiqi, Multiple error detection and correction based on redundant residue number systems, IEEE Transactions on Communications, (56) 3 (2008), pp. 325-330.

[17] A.Omondi, B. Premkumar, Residue Number System: Theory and Implementation, Imperial College Press, 2007, 296 p.

[18] I.Y. Akushskiy, D. I. Yuditskiy, Machine Arithmetics in Residue Number System, Moscow, Soviet Radio, 1968, 460 p. (in Russian).

[19] I. Y. Akushskiy, I. T.Pak, Questions of errorcorrecting coding position-independent code, Problems of Cybernetics, (208) (1977), pp. 3656. (in Russian).

[20] Hu Zhengbing, V. Yatskiv, A. Sachenko, Increasing the data transmission robustness in WSN using the modified error correction codes on residue number system, Elektronika ir Elektrotechnika, (21) 1 (2015), pp. 76-81.

[21] A. Sikora, Web2.0 technology for an embedded web-based gateway platform for spatially distributed wireless networks, International Journal of Computing, (9) 1 (2010), pp. 31-36.

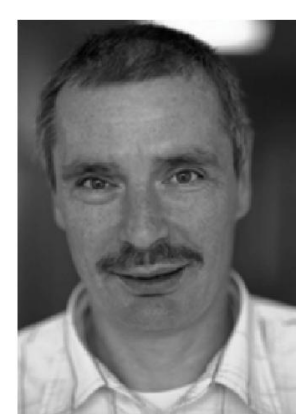

Prof. Dr. Jürgen Sieck received his degree in mathematics in 1981 and his $\mathrm{PhD}$ in Computer Science in 1989 from the Humboldt University Berlin, Germany. Now he is the senior researcher at the research group "Informations- und Kommunikationsanwendungen" (INKA)

and professor for computer sciences with a specialisation on algorithms, multimedia and mobile application for the degree programme Applied Computer Science at the University of Applied Sciences HTW Berlin. Previously, he was visiting lecturer at Monash University Melbourne (Australia), Yuanpei University Hsinchu (Taiwan) and at the University of Cape Town (South Africa) and was visiting researcher at the International Computer Science Institute in Berkley (USA), Polytechnic of Namibia and at Old Dominion University Norfolk in Virginia (USA). In February 2013 he was awarded an honorary doctorate from Odessa National Polytechnic University (Ukraine). 


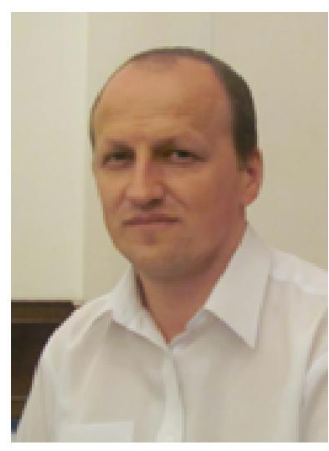

Vasyl Yatskiv. Eng. Automation of technological processes. Ivano-Frankivsk Technical Univercity of Oil and Gas, Ukraine (1996). Ph.D. of Computers, system and network. Lviv Polytechnic Univercity, Ukraine (2001).

Associate Professor of Department of Specialized Computing Systems at Fa-

culty of Computer Information Technologies of Ternopil National Economic University.

His main Areas of Research Interest are Wireless Sensor Networks, Residue Number System, Correcting Codes.

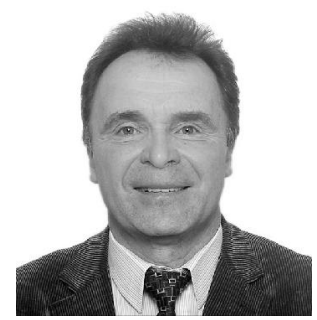

Anatoly Sachenko is Professor and Head of the Department of Information Computing Systems and Control and Research advisor of the Research Institute for Intelligent Computer Systems, Ternopil National Economic University.

$\mathrm{He}$ earned his B.Eng. Degree in Electrical Engineering at L'viv Polytechnic Institute in1968 and his PhD Degree in Electrical Engineering at L'viv Physics and Mechanics Institute in 1978 and his Doctor of Technical Sciences Degree in Electrical and Computer Engineering at Leningrad Electrotechnic Institute in 1988. Since 1991 he has been Honored Inventor of Ukraine, since 1993 he has been IEEE Senior Member.

His main Areas of Research Interest are Implementation of Artificial Neural Network, Distributed System and Network, Parallel Computing, Intelligent Controllers for Automated and Robotics Systems. He has published over 530 papers in areas above.

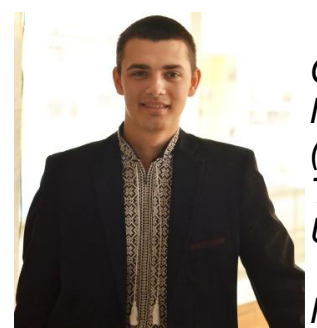

Taras Tsavolyk, master in Computer Engineering. Ternopil National Economic University (2014). Ph.D. student of the Ternopil National Economic University, Ukraine (2014).

His main Areas of Research Interest are Wireless Sensor Networks, Residue Number System, Correcting Codes. 